\title{
Phase-field investigation of lithium electrodeposition under different applied overpotentials and operating temperatures
}

\author{
Joonyeob Jeon, ${ }^{1,2}$ Gil Ho Yoon, ${ }^{2}$ Tejs Vegge, ${ }^{1}$ and Jin Hyun Chang ${ }^{1,3, a)}$ \\ 1) Department of Energy Conversion and Storage, Technical University of Denmark, \\ DK-2800 Kgs. Lyngby, Denmark \\ ${ }^{2)}$ School of Mechanical Engineering, Hanyang University, Seoul, \\ South Korea \\ 3) PhaseTree ApS, Copenhagen, Denmark
}

(Dated: 21 December 2021)

Despite the high promise, the commercialization of Li-metal-based batteries has been hampered due to the formation of dendrites that lead to mechanical instability, energy loss and eventual internal short circuits. The mechanism of dendrite formation and the strategies to suppress their growth have been studied intensively. However, the effect of applied overpotential and operating temperature on dendrite growth remains to be fully understood. Here, we elucidate the correlation of overpotential and temperature with the surface modulation during electrodeposition using phase-field simulations. We identify an optimal operating temperature of half-cell consisting of a $\mathrm{Li}$ metal anode and $1 \mathrm{M} \mathrm{LiPF}_{6}$ in $\mathrm{EC}: \mathrm{DMC}(1: 1)$, which increases gradually as the overpotential increases. The investigation reveals that the temperature dependence identified in the simulations and experiments often disagree because they are primarily conducted in galvanostatic and potentiostatic conditions, respectively. The temperature increase under potentiostatic conditions increases the induced current while it decreases the induced overpotential under galvanostatic conditions. Therefore, the analysis and comparison of temperature-dependent characteristics must be carried out with care.

\footnotetext{
${ }^{a)}$ Electronic mail: jchang@dtu.dk
} 


\section{INTRODUCTION}

There is an urgent need for energy storage devices that perform beyond current stateof-the-art Li-ion batteries for various technology sectors, including transportation and grid energy storage. Lithium ( $\mathrm{Li}$ ) metal is considered to be one of the most promising electrode materials for next-generation battery technologies and is used as an anode material for Limetal, ${ }^{1-3}$ Li-sulfur, ${ }^{4-6}$ and Li-air batteries. ${ }^{7-10}$ Li metal is investigated in many upcoming battery technologies due to its merits, including a very high energy density of $3680 \mathrm{~mA} \mathrm{~h} \mathrm{~g}^{-1}$ and a low redox potential of $-0.304 \mathrm{~V}$ vs the standard hydrogen electrode and mass density of $0.534 \mathrm{~g} \mathrm{~cm}^{-3} \cdot{ }^{11,12}$ These merits make Li a particularly attractive electrode material; the use of Li metal could substantially increase the energy density of batteries. ${ }^{13}$

Despite advantages, the commercialization of the battery technologies based on Li metal is hindered in part by the formation and growth of dendrites on the Li surface upon cycling. ${ }^{14}$ The Li metal is used as an anode in the Li-metal-based batteries, which means that electrodeposition and electrodissolution processes take place at the Li-metal surface during charge and discharge, respectively. The non-uniform electrodeposition and electrodissolution processes across the Li surface are the root cause of the formation and growth of the dendrites, ${ }^{1,11}$ and they are of great concern for battery applications due to their detrimental effect on the performance and safety of the batteries. Dendrites can cause an internal shortcircuit when they penetrate the separator, causing a thermal runaway and failure. Although not detrimental, the dendrites can also detach from the electrode during electrodissolution, leading to performance degradation as the metallic debris do not participate in electrochemical reactions. Consequently, understanding and determining safe operating conditions that suppress the formation and growth of dendrites are important for the commercialization of the next-generation batteries that incorporate Li metals.

It is by no means straightforward to accurately predict the morphology and evolution of dendrite as they are nonlinear processes that depend on many factors such as impurity/defect concentration, applied potential/current, pressure and operating temperature, to list a few. ${ }^{12,15-17}$ A number of experimental and computational studies have been carried out to understand the underlying mechanisms and correlations between the growth rate and battery operating conditions. ${ }^{15,16,18-20}$ In particular, the growth of dendrite under different applied current densities (galvanostatic) ${ }^{21,22}$ and electric potentials (potentiostatic) ${ }^{16}$ across 
the interface was investigated. It was experimentally observed in several reports that increasing the applied current or potential promotes the growth of the dendrites when the other conditions are unchanged. ${ }^{11,20,23,24}$ The temperature dependence is often overlooked in the analyses, although it is a critical factor to consider. Commercial batteries are expected to have a wide operating temperature range, and thus, the correlation between the dendrite evolution and the operating temperature needs to be established to ensure the safe and effective operation of batteries. A recent study based on in situ optical microscopy and ex situ scanning electron microscopy (SEM) revealed a dramatic change in the dendrite growth ${ }^{20}$; under fixed current density conditions, elevating the operating temperature was observed to result in a larger Li nuclei size and a lower nucleation density. The elevated temperature from internal heating is reported to smooth the surface due to extensive surface migration. ${ }^{25}$

The phase-field method ${ }^{26,27}$ is one of the most popular computational methods for modeling the dendrite growth as it is well-suited to simulate the evolution of the interface between two dissimilar materials. The Li dendrite growth is a nonlinear process that depends on various factors such as electrochemical reactions, applied overpotential, operating temperature and Li-ion concentration in the electrolyte. ${ }^{22,28}$ Consequently, nonlinear phase-field models that directly include the contributions of these factors have been used to predict the dendrite evolution. ${ }^{17-19,29}$ The impact of applied overpotential across the interface on the dendrite evolution has been studied in the past ${ }^{17,18}$ as it is an externally controllable factor that directly affects dendrite growth mechanism and rate. Hong and Viswanathan ${ }^{17}$ inves- $^{-}$ tigated the evolution of the Li electrode when three different overpotentials (i.e., $-0.32 \mathrm{~V}$, $-0.45 \mathrm{~V}$ and $-0.50 \mathrm{~V}$ ) were applied across the electrode-electrolyte interface. They found that the Li ions get consumed at a higher rate through electrochemical reactions under a higher overpotential. The electrodeposition process is more prone to become transportlimited as the Li ions at the interface become depleted. The presence of the small peaks due to the inhomogeneity boosts the ion transport in its vicinity due to the migration from the surrounding valley regions, thereby increasing the electrochemical reaction rate near the peak while further depleting the Li-ion concentration in valleys. ${ }^{17}$ Interestingly, Hong and Viswanathan ${ }^{17}$ argued that the key feature that distinguishes the low-overpotential regime (no dendrite formation) from the high-overpotential regime (dendrite formation) is the concentration of $\mathrm{Li}$ ions at the interface relative to that of the bulk; the Li concentration at the interface is higher than that of the bulk value when the overpotential is low, while the 
opposite is true when the overpotential is high.

Only a limited number of computational studies investigated the thermal effect on the dendrite evolution. Recent works ${ }^{15,16}$ include derivation of temperature field using heat transfer model and coupling it with diffusion coefficients. This elegant approach is capable of not only including the change in the ambient temperature but also the internal heat generation, convection and radiation. A simpler model that substitutes different temperature values in the governing equations without any coupling term has also been used to investigate the electrodeposition of zinc. ${ }^{30}$ Gao and Guo ${ }^{31}$ accounted for heat generation and diffusion in their temperature field to investigate the internal temperature distribution. However, the state-of-the-art phase-field models only account for the local temperature effect on diffusivity values, and comprehensive inclusion of the thermal effect on other physicochemical parameters remains missing.

This work aims to fully integrate the thermal effects on various aspects of the nonlinear phase-field model to assess the contribution of the operating temperature and applied overpotential to the dendrite formation and growth rate. We included the temperature dependence of the electrode conductivity, electrolyte conductivity, surface tension, exchange current density, as well as the Li-ion diffusivity in the electrolyte. The electrodeposition process is simulated at varying operating temperatures and applied overpotentials in order to assess the contribution of the two, and the resulting morphology evolution is analyzed in terms of the tortuosity and the maximum height of the dendrite when the same amount of Li ions are deposited onto the electrode. Our results show that induced current across the interface increases when the temperature is elevated, and a direct comparison of temperature dependence based on galvanostatic and potentiostatic results should be avoided. The comparison of surface modulation after depositing the same amount of Li under different temperatures and applied overpotentials revealed that the dendrite growth rate as a function of the amount of deposited Li is similar across cases. A critical factor to consider is the onset point at which dendrites start to form, which is determined via setting threshold criteria for the dendrite height and tortuosity. Using the developed phase-field model, we have identified that increasing the magnitude of overpotential lowers the onset point and determined an optimal operating temperature under different values of applied overpotential. 


\section{METHODOLOGY}

\section{A. Model overview}

We investigated a half-cell system consisting of $\mathrm{Li}$ metal anode and $1 \mathrm{M} \mathrm{LiPF}_{6}$ in EC:DMC(1:1). The two-dimensional simulation cell has a dimension of $200 \mu \mathrm{m}$ by $200 \mu \mathrm{m}$; the anode has an initial thickness of $20 \mu \mathrm{m}$ while the remaining $180 \mu \mathrm{m}$ is occupied by the electrolyte, as shown in Fig. 1. The $\mathrm{Li}^{+}$ion from the electrolyte approaches the Li-metal surface during the electrodeposition process, reacting with the electrons in the electrode and becoming reduced to Li atoms. The electrodeposition process can be described using a simple chemical reaction:

$$
\mathrm{Li}^{+}+\mathrm{e}^{-} \longrightarrow \mathrm{Li}
$$

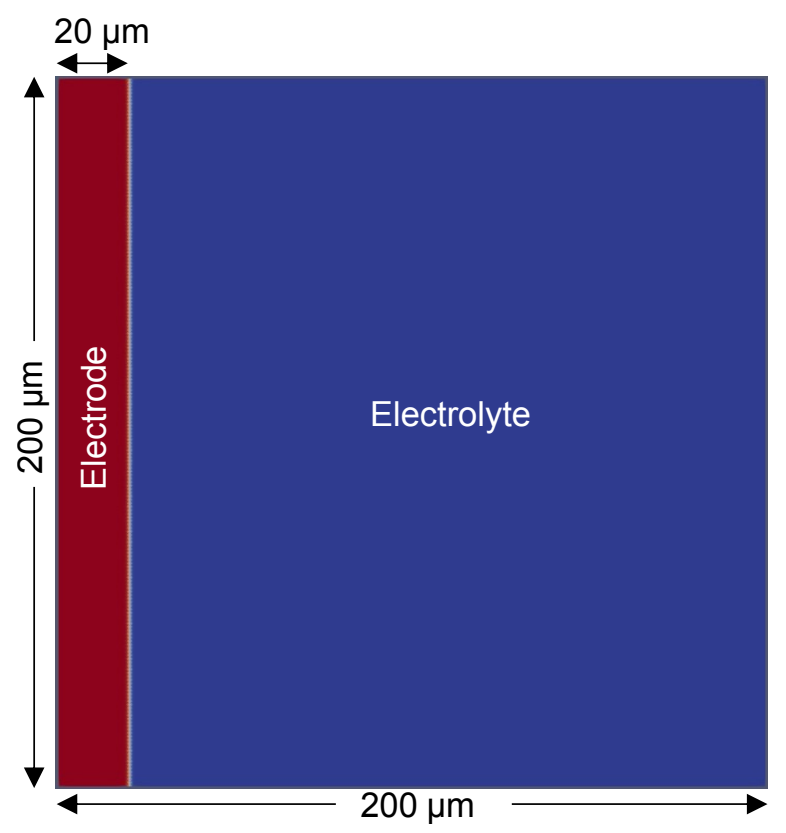

FIG. 1. Initial geometry of the simulation cell before electrodeposition.

The two phases of the system — Li metal anode and electrolyte — are distinguished using the order parameter, $\xi$. The order parameter is a continuous parameter that represents the phase of the system, and it ranges from 0 to $1 . \xi=0$ corresponds to the electrolyte phase while $\xi=1$ corresponds to the Li metal phase. The interface between the electrode and electrolyte has a finite thickness where the value of $\xi$ lies between 0 and 1 . All of the reported results are generated using the phase-field simulation module of PhaseTree. ${ }^{32}$ 


\section{B. Allen-Cahn equation}

A description of the free energy of the interface is important for constructing a phasefield model. The interfacial free energy of the Ginzburg-Landau type ${ }^{26}$ is expressed using the multi-well potential and gradient coefficient energy, ${ }^{18,19,28,29,31}$ which makes the sharp interface energy with the smooth profiles of the phase fields. The system considered for this work is represented using a double-well potential with two equilibrium states, $\xi=0$ and $\xi=1$. The interfacial free energy, $U$, is written as

$$
U=\int \frac{\kappa}{2}|\nabla \xi|^{2}+g(\xi) d V,
$$

where $\frac{\kappa}{2}|\nabla \xi|^{2}$ and $g(\xi)$ are the terms describing the gradient energy density and double-well

potential, respectively. The gradient energy term models the diffusion process that smooths out the order parameter while the double-well potential term counteracts such smoothing by separating the values through the potential barrier. ${ }^{26} \kappa$ is a gradient coefficient defined as $\kappa=\frac{3}{2} \gamma \delta$, where $\gamma$ is the surface tension and $\delta$ is the interface thickness. The doublewell potential is written as $g(\xi)=\omega \xi^{2}\left(1-\xi^{2}\right)$, where the barrier height, $\omega$, is defined as $\omega=12 \frac{\gamma}{\delta} \cdot 18,19,26$

The temporal evolution of the order parameter is related to the interfacial free energy by

$$
\frac{\partial \xi}{\partial t}=-L_{\sigma}\left(\frac{\partial U}{\partial \xi}\right)
$$

where $L_{\sigma}$ is the interface mobility. Substituting Eq. (2) into $U$ in Eq. (3) leads to a wellknown Allen-Cahn equation,

$$
\frac{\partial \xi}{\partial t}=-L_{\sigma}\left(g^{\prime}(\xi)-\kappa \nabla^{2} \xi\right) .
$$

Allen-Cahn equation is the first component describing the temporal evolution of the electrode-electrolyte interface during electrodeposition.

\section{Modified Butler-Volmer equation}

The description of the temporal evolution thus far does not include the change in the energy due to the electrochemical reactions. The electrochemical reaction kinetics is described using a modified Butler-Volmer equation, which can be written as

$$
\frac{\partial \xi}{\partial t}=-L_{\eta} h^{\prime}(\xi)\left\{\exp \left[\frac{(1-\alpha) n F \eta_{\alpha}}{R T}\right]-\frac{c_{\mathrm{Li}^{+}}}{c_{0}} \exp \left[\frac{-\alpha n F \eta_{\alpha}}{R T}\right]\right\} .
$$


$L_{\eta}=\frac{V_{m} \gamma}{F \kappa} i_{0}$ is the electrochemical reaction kinetic coefficient, where $V_{m}$ is the molar volume of $\mathrm{Li}$ and $i_{0}$ is the exchange current density. $h(\xi)$ is the interpolating function that is generally used in phase-field modeling, and the most commonly used function is $h(\xi)=$ $\xi^{3}\left(6 \xi^{2}-15 \xi+10\right)$, which is also used in this work. While satisfying the condition of smoothly interpolating $\xi$ from 0 to 1,33 the function also has its derivative, $h^{\prime}(\xi)=30 \xi^{2}(1-$ $\xi)^{2}$, that limits the electrochemical reactions to take place only at the interface as $h^{\prime}$ becomes zero when the value of $\xi$ approaches 0 or 1 . $F$ is Faraday's constant $\left(96485 \mathrm{C} \mathrm{mol}^{-1}\right), R$ is the gas constant $\left(8.314 \mathrm{~J} \mathrm{~mol}^{-1} \mathrm{~K}^{-1}\right.$ ), $\alpha$ is the charge transfer coefficient (set to 0.5 in this work), $n$ is the number of electrons transferred in the reaction ( 1 for Li electrodeposition as described in Eq. (1)) and $T$ is the temperature in kelvin. The activation overpotential, $\eta_{\alpha}$, is defined as $\eta_{\alpha}=\phi-E_{0}$, where $\phi$ is the applied overpotential and $E_{0}$ is the standard equilibrium half cell potential, which is set to zero. $c_{0}$ and $c_{\mathrm{Li}^{+}}$, respectively, are the initial and local Li-ion molar ratios of the electrolyte, where the initial molar ratio corresponds to the molar ratio of the bulk electrolyte that serves as the baseline for assessing the local molar ratio near the interface.

The overall temporal evolution of the order parameter (i.e., the evolution of surface morphology) can be described via the superposition of Eq. (4) and Eq. (5), ${ }^{17-19,28}$ which is expressed as

$$
\frac{\partial \xi}{\partial t}=-L_{\sigma}\left(g^{\prime}(\xi)-\kappa \nabla^{2} \xi\right)-L_{\eta} h^{\prime}(\xi)\left\{\exp \left[\frac{(1-\alpha) n F \eta_{\alpha}}{R T}\right]-\frac{c_{\mathrm{Li}^{+}}}{c_{0}} \exp \left[\frac{-\alpha n F \eta_{\alpha}}{R T}\right]\right\} .
$$

The expression in Eq. (6) does not account for any perturbation in the system due to surface defects and thermal variations that may trigger the formation of the dendrite nucleus. Therefore, we added Langevin noise with a magnitude of 0.04 in Eq. (6) to represent the random perturbation.

\section{Modified diffusion equation}

The temporal evolution of chemical potential, $\mu$, derived from the mass conservation law is written as ${ }^{17,19}$

$$
\frac{\partial \mu}{\partial t}=\frac{1}{\chi}\left[\nabla \cdot \frac{D c_{\mathrm{Li}^{+}}}{R T}(\nabla \mu+n F \nabla \phi)-\frac{\partial h(\xi)}{\partial t}\left(c^{s} \frac{C_{m}^{s}}{C_{m}^{l}}-c^{l}\right)\right],
$$


where the susceptibility factor, $\chi$, is defined as

$$
\chi=\frac{\partial c^{l}}{\partial \mu}[1-h(\xi)]+\frac{\partial c^{s}}{\partial \mu} h(\xi) \frac{C_{m}^{s}}{C_{m}^{l}} .
$$

$C_{m}^{l}$ and $C_{m}^{s}$ are site densities of the liquid (electrolyte) and solid (electrode) phases, respectively. Similarly, $c^{l}$ and $c^{s}$ respectively represent the molar ratios of solid and liquid phases and are related to the chemical potential of the system by

$$
c^{l, s}=\frac{\exp \left(\frac{\mu-\epsilon^{l, s}}{R T}\right)}{1+\exp \left(\frac{\mu-\epsilon^{l, s}}{R T}\right)} .
$$

Here, $\epsilon^{l, s}$ is the difference in the chemical potential of Li species with respect to that of the neutral components at the initial equilibrium. The local Li-ion molar ratio of the electrolyte is related to the molar ratio of the liquid phase via $c_{\mathrm{Li}^{+}}=c^{l}(1-h(\xi))$.

\section{E. Charge conservation equation}

The system is electrically neutral and its charge conservation is described using Poisson's equation, which is written as

$$
\nabla \sigma \nabla \phi=n F C_{m}^{s} \frac{\partial \xi}{\partial t}
$$

$\sigma$ is the effective conductivity and is related to the conductivity of the electrode, $\sigma^{s}$, and electrolyte, $\sigma^{l}$, using the interpolation function as

$$
\sigma=\sigma^{s} h(\xi)+\sigma^{l}(1-h(\xi))
$$

\section{F. Temperature dependence of the parameters}

The description of the phase-field model thus far provides an overview without the influence of the temperature of the system. The impact of the temperature is directly included with the temperature term, $T$, in cases such as the modified Butler-Volmer expression in Eq. (6). However, most of its effect is reflected through the temperature dependence of the physicochemical parameters. The temperature dependence of the parameters - exchange current density, ionic diffusivity, electrode and electrolyte conductivity and surface tensionare shown in Fig. 2. 


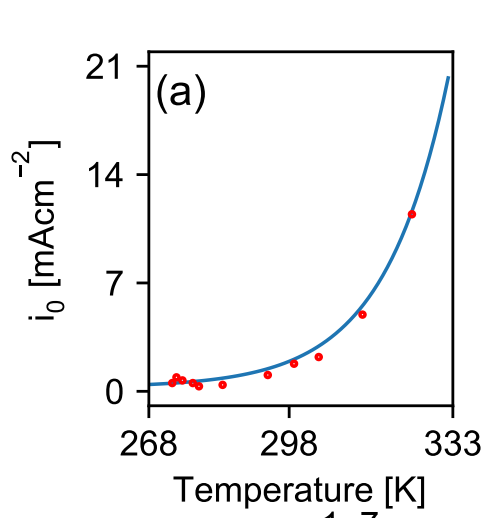

Temperature $[\mathrm{K}]$

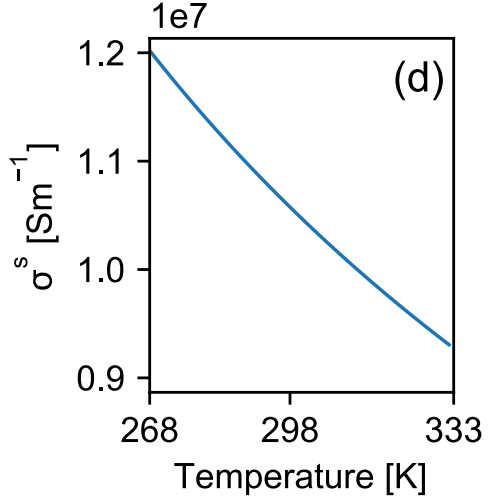

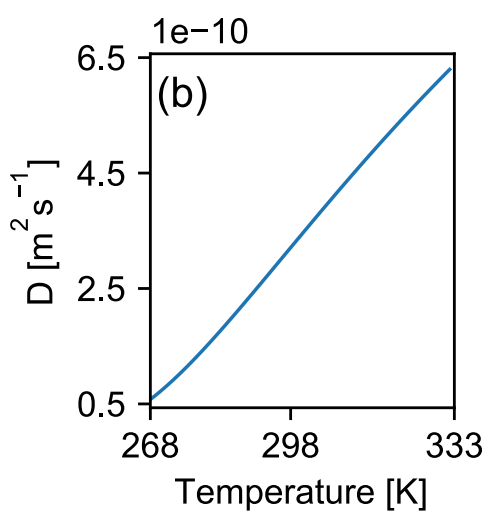
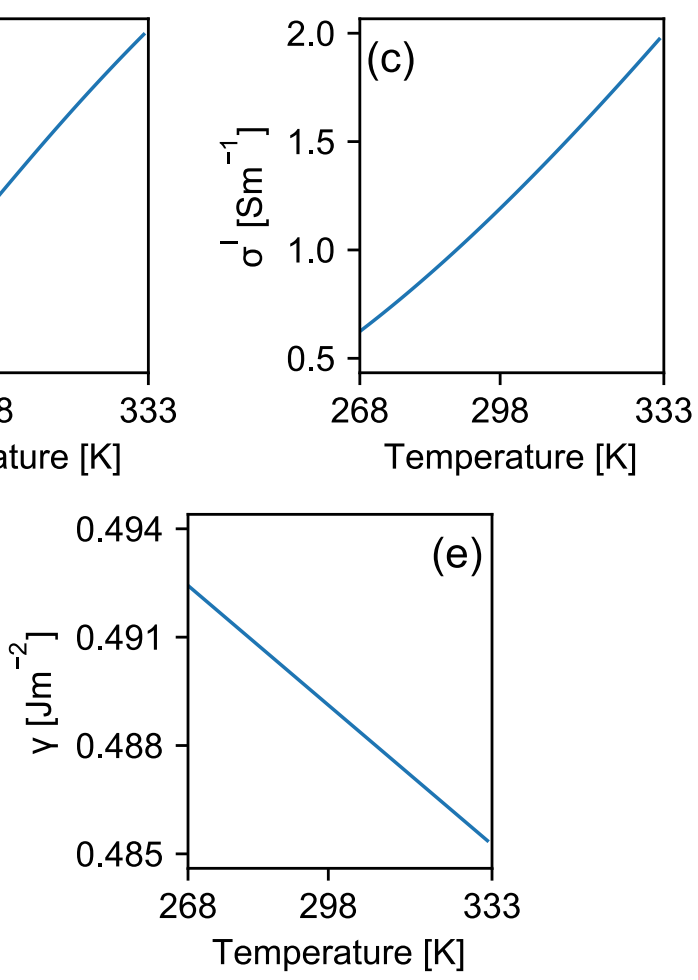

FIG. 2. Temperature-dependent values of (a) exchange current density, ${ }^{34}$ (b) ionic diffusivity of the electrolyte, ${ }^{35}$ (c) conductivity of electrolyte, ${ }^{35}$ (d) conductivity of electrode ${ }^{36}$ and (e) surface tension of electrode ${ }^{37}$ for the temperature ranging from $268 \mathrm{~K}$ to $333 \mathrm{~K}$.

The exchange current density, $i_{0}$, across the Li metal anode and $1 \mathrm{M} \mathrm{LiPF}_{6}$ in $\mathrm{EC}: \mathrm{DMC}(1: 1)$ is taken from the work of Hess ${ }^{34}$ who reported the experimentally measured values at temperatures between $253.55 \mathrm{~K}$ and $344.35 \mathrm{~K}$. The reported values are shown as red dots in Fig. 2(a). The experimental data were fitted using an exponential regression model (shown as a blue line). The fitted exchange current in $\mathrm{mA} \mathrm{cm}^{-2}$ is expressed as

$$
i_{0}(T)=6.5 \times 10^{-10} \exp (0.0727 T)+0.25
$$

The temperature dependence of the ionic diffusivity was obtained from the experimental measurements of Valøen and Reimers ${ }^{35}$, who reported that the ionic diffusivity, $D$, in $\mathrm{m}^{2} \mathrm{~s}^{-1}$ is

$$
\log _{10} D(T)=-8.65-\frac{54}{(T-234)}
$$

for $T$ between $263 \mathrm{~K}$ and $333 \mathrm{~K}$.

The conductivity values are also extracted from the experimentally obtained values at 
varying temperatures. The electrolyte conductivity, $\sigma^{l}$, in $\mathrm{S} \mathrm{m}^{-1}$ is expressed as ${ }^{35}$

$$
\sqrt[2]{10 \cdot \sigma^{l}(T)}=\sum_{i=0}^{2} \sum_{j=0}^{2} k_{i j} \cdot T^{j}
$$

for temperature between $263 \mathrm{~K}$ and $333 \mathrm{~K}$. The values of $k_{i j}$ for all values of $i$ and $j$ are shown in Table I. The resistivity, $R$, of Li metal in $\Omega \mathrm{m}$ is related to the temperature as

$$
\begin{aligned}
\log _{10} R(T)= & -7.8425+2.314 \log _{10} \frac{T}{92.295}-1.962\left(\log _{10} \frac{T}{92.295}\right)^{2} \\
& +1.127\left(\log _{10} \frac{T}{92.295}\right)^{3}
\end{aligned}
$$

for temperature ranging from $92.295 \mathrm{~K}$ to $453.6 \mathrm{~K}$ according to the report by $\mathrm{Chi}^{36}$. The conductivity of the Li metal electrode, $\sigma^{s}$, in $\mathrm{S} \mathrm{m}^{-1}$ is the reciprocal of the resistivity,

$$
\sigma^{s}(T)=\frac{1}{R(T)}
$$

Another parameter that is known to have temperature dependence is the surface tension of Li metal, $\gamma$. Its value is reported ${ }^{37}$ to be

$$
\gamma(T)=0.472+1.1034 \times 10^{-4}(453.15-T)
$$

for temperature between $0 \mathrm{~K}$ and $453.15 \mathrm{~K}$, where its value is in $\mathrm{J} \mathrm{m}^{-2}$. The rest of the parameters that do not depend on temperature are summarized in Table II.

TABLE I. $k_{i j}$ for calculating the electrolyte conductivity. Values are taken from ref. 35.

\begin{tabular}{cccc}
\hline \hline$k_{i j}$ & $j=0$ & $j=1$ & $j=2$ \\
\hline$i=0$ & -10.5 & 0.0740 & $-6.96 \times 10^{-5}$ \\
$i=1$ & 0.668 & -0.0178 & $2.80 \times 10^{-5}$ \\
$i=2$ & 0.494 & $-8.86 \times 10^{-4}$ & 0 \\
\hline \hline
\end{tabular}

Finally, the chemical potential difference between the Li and neutral species at the initial equilibrium, $\epsilon^{l, s}$, is estimated using the initial molar ratio of Li species, $c^{0 l, 0 s}$. The approximation is written as $\epsilon^{l, s}=-R T \ln \frac{c^{0 l, 0 s}}{1-c^{0 l, 0 s}}$ based on the work of Cogswell ${ }^{19}$. Since the initial molar ratios $c^{0 l}$ and $c^{0 s}$ are 0.067159 and 0.999999 , respectively, $\epsilon^{l}=2.631 R T$ and $\epsilon^{s}=-13.8 R T$. 
TABLE II. Constant parameters of the phase-field model and their normalized values.

\begin{tabular}{|c|c|c|c|c|}
\hline Symbol & Name & Value & Normalized value & Reference \\
\hline$L_{\sigma}$ & Interfacial mobility & $2.5 \times 10^{-6} \mathrm{~m}^{3} \mathrm{~J}^{-1} \mathrm{~s}^{-1}$ & 6.25 & 18 \\
\hline$n$ & Num. of electrons transferred & 1 & 1 & - \\
\hline$\delta$ & Interface thickness & $1 \mu \mathrm{m}$ & 1 & 17 \\
\hline$\alpha$ & Transfer coefficient & 0.5 & 0.5 & 38 \\
\hline$C_{m}^{s}$ & Site density of electrode & $7.64 \times 10^{4} \mathrm{~mol} \mathrm{~m}^{-3}$ & 76.4 & 15,39 \\
\hline$C_{m}^{l}$ & Site density of electrolyte & $1.44 \times 10^{4} \mathrm{~mol} \mathrm{~m}^{-3}$ & 14.4 & 17 \\
\hline$c^{0 l}$ & Initial Li electrolyte molar ratio & 0.067159 & 0.067159 & 17 \\
\hline$c^{0 s}$ & Initial Li electrode molar ratio & 0.999999 & 0.999999 & Estimated \\
\hline
\end{tabular}

\section{G. Numerical settings}

The phase-field model solves the temporal and spatial evolution of three parameters: the order parameter, chemical potential and electric overpotential. Each of these parameters needs initial and boundary conditions defined in addition to the governing equations described through Eq. (6), Eq. (7) and Eq. (10). The boundary conditions of the three parameters are shown in Table III.

The initial geometry shown in Fig. 1 is represented via the distribution of order parameter at $t=0 \mathrm{~s}$,

$$
\xi(x, y)=\frac{1-\tanh [2(x-20)]}{2},
$$

which represents the electrode thickness of $20 \mu \mathrm{m}$ with a smooth transition from the electrode to the electrolyte at the interface. The initial distribution of electric potential is set as

$$
\phi(x, y)=\phi_{\text {applied }} \xi(x, y)
$$

such that the electrode and electrolyte has the potential of $\phi_{\text {applied }}$ and 0 in the beginning of the simulation. The chemical potential was initially set to zero across the entire domain. The $200 \mu \mathrm{m} \times 200 \mu \mathrm{m}$ domain is represented with a $200 \times 200$ crossed mesh, and it is solved using Newton's iterative method with adaptive time steps. The distribution of order parameter, chemical potential and electric overpotential are saved at $1 \mathrm{~s}$ time interval. The normalization factors of length, time, temperature, mol, energy and conductance are $1 \mu \mathrm{m}$, $1 \mathrm{~s}, 1 \mathrm{~K}, 1 \times 10^{-15} \mathrm{~mol}, 2.5 \times 10^{-12} \mathrm{~J}$ and $1 \times 10^{-6} \mathrm{~S}$, respectively. 
TABLE III. Boundary conditions used for the phase-field model.

\begin{tabular}{|c|c|c|c|}
\hline Parameter & Boundary & Boundary condition type & Value \\
\hline \multirow{4}{*}{$\xi$} & $x=0 \mu \mathrm{m}$ & Dirichlet & 1 \\
\hline & $x=200 \mu \mathrm{m}$ & Dirichlet & 0 \\
\hline & $y=0 \mu \mathrm{m}$ & Neumann & 0 \\
\hline & $y=200 \mu \mathrm{m}$ & Neumann & 0 \\
\hline \multirow{4}{*}{$\mu$} & $x=0 \mu \mathrm{m}$ & Dirichlet & 0 \\
\hline & $x=200 \mu \mathrm{m}$ & Dirichlet & 0 \\
\hline & $y=0 \mu \mathrm{m}$ & Neumann & 0 \\
\hline & $y=200 \mu \mathrm{m}$ & Neumann & 0 \\
\hline \multirow{4}{*}{$\phi$} & $x=0 \mu \mathrm{m}$ & Dirichlet & $\phi_{\text {applied }}^{*}$ \\
\hline & $x=200 \mu \mathrm{m}$ & Dirichlet & 0 \\
\hline & $y=0 \mu \mathrm{m}$ & Neumann & 0 \\
\hline & $y=200 \mu \mathrm{m}$ & Neumann & 0 \\
\hline
\end{tabular}

* the value of overpotential applied across the interface.

\section{H. Assessment of the surface modulation}

The tree- or needle-like patterns ${ }^{40,41}$ of dendrites are not only found in phase-field simulations but also in experimental observations. ${ }^{42}$ As the shapes and patterns of dendrites can vary under different operating conditions, a quantitative assessment scheme is needed to evaluate the surface modulation to determine the presence of dendrites and the extent to which they have grown. In this work, we are using the height of the dendrite and the tortuosity of the surface to quantitatively describe the surface modulation. The dendrite height is defined as a difference between the average height of the Li metal and the maximum height of its peaks; it is a measure of the peak height with respect to that of the average. Tortuosity of the surface, on the other hand, describes the "roughness" of the surface upon electrodeposition and is defined as the ratio between the length of the curved path and the straight path connecting the two endpoints. In other words, tortuosity is defined as $\tau=\frac{l_{c}}{l_{s}}$, where $l_{c}$ and $l_{s}$ correspond to the length of curved and straight paths, respectively. A perfectly smooth surface will have $\tau=1$, and the roughness of the surface will increase the tortuosity. 
The effects of dendrite height and tortuosity are illustrated in Fig. 3. It is seen from Fig. 3(a) that assessing the surface morphology based on the dendrite height alone can be misleading. A hill-like shape with a gradual change in height leads to a low tortuosity value, and such a morphology does not represent a scenario of dendrite formation. On the contrary, a rough surface free of any noticeable dendrite has a high tortuosity with low dendrite height as shown in Fig. 3(b). The first two cases demonstrate that neither the height nor the tortuosity should be used alone to quantitatively evaluate the surface morphology. A schematic shown in Fig. 3(c) has a needle-like extrusion on otherwise perfectly smooth surface. The tortuosity is noticeably higher than that of the hill-like pattern even in the presence of a single needle-like dendrite, and the tortuosity becomes significantly higher in the presence of multiple dendrites as shown in Fig. 3(d). Therefore, we use both the dendrite height and tortuosity to assess the presence of the dendrite, i.e., both the dendrite height and tortuosity should exceed their threshold values for the surface to be classified to have dendrites.
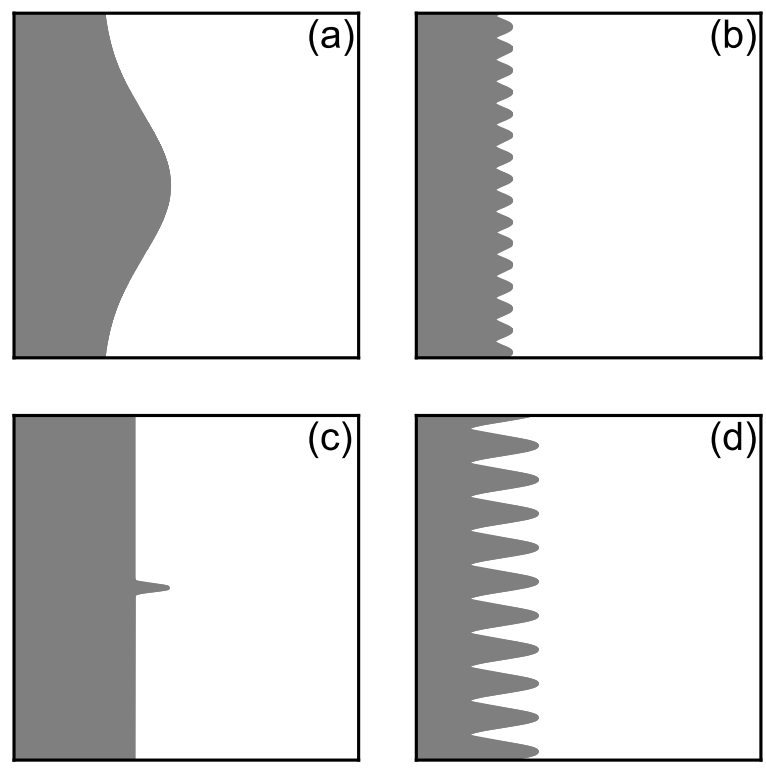

FIG. 3. Schematics of surfaces with different dendrite height and tortuosity. (a) height $=20 \mu \mathrm{m}$, tortuosity $=1.08,(\mathrm{~b})$ height $=5 \mu \mathrm{m}$, tortuosity $=1.94,(\mathrm{c})$ height $=20 \mu \mathrm{m}$, tortuosity $=1.16,(\mathrm{~d})$ height $=20 \mu \mathrm{m}$, tortuosity $=4.26$. 


\section{RESULTS AND DISCUSSIONS}

The temperature and overpotential are two control parameters considered in this study. The rest of the system parameters are kept constant, except for their dependence on the temperature. The considered temperature range is from $268 \mathrm{~K}$ to $333 \mathrm{~K}$ with a $5 \mathrm{~K}$ increment. The temperature range was selected based on the availability of experimental reports that provide the necessary physicochemical parameters for the phase-field model; a temperature range is selected such that all temperature-dependent parameter values are available. The range of applied overpotential was determined based on the work of Hong and Viswanathan ${ }^{17}$, which determined that dendrites form at $-0.45 \mathrm{~V}$ and $-0.50 \mathrm{~V}$ but not at $-0.32 \mathrm{~V}$ at room temperature. Based on their report, this work investigates the effect of overpotential by considering the range between $-0.30 \mathrm{~V}$ and $-0.44 \mathrm{~V}$ with a $0.02 \mathrm{~V}$ increment.

\section{A. Predicting dendrite formation using the Li-ion concentration profile}

One crucial finding of Hong and Viswanathan ${ }^{17}$ was that dendrite formation could be predicted early in the electrodeposition simulation based on the Li-ion concentration profile. In particular, it was observed that dendrites form when the Li-ion concentration at the interface falls below that of the bulk. For the cases where no surface modulation is observed, on the other hand, the Li-concentration at the interface was mostly higher than that of the bulk during electrodeposition, although it oscillates above and below the bulk value. The difference was understood as a result of direct competition between the ionic transport and electrochemical reaction. ${ }^{17,43}$ The surface grows uniformly when the process is reaction limited (i.e., the reaction rate is slower than the transport rate), and the interface has the accumulation of $\mathrm{Li}$ ions. In contrast, a transport-limited process leads to a depletion of ions at the interface that causes an inhomogeneity of electrochemical reactions at the surface, which in turn promotes the dendrite formation. The two scenarios are illustrated in Fig. 4. We investigated the generalizability of their observation as the first step since the original investigation was limited to room temperature $(300 \mathrm{~K})$. More specifically, we demonstrate that the Li-ion concentration profiles early in the simulation can be used to predict the dendrite formation later in the electrodeposition process as depicted in Fig. 4 for different 
applied overpotentials and operating temperatures.

(a)

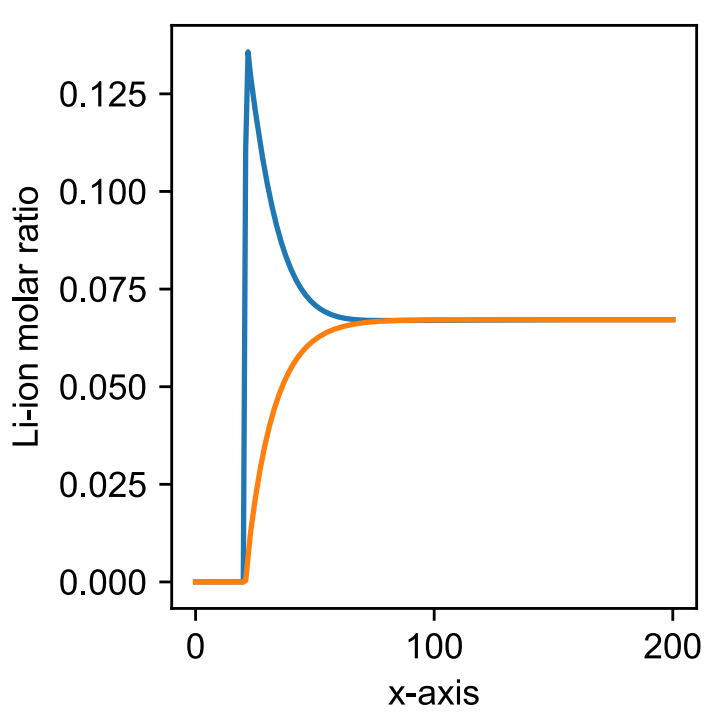

(b)

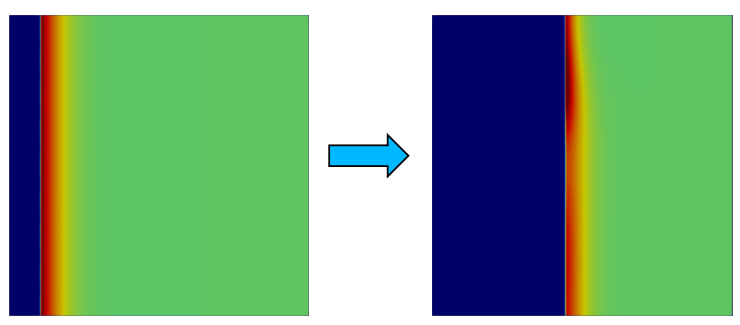

(c)

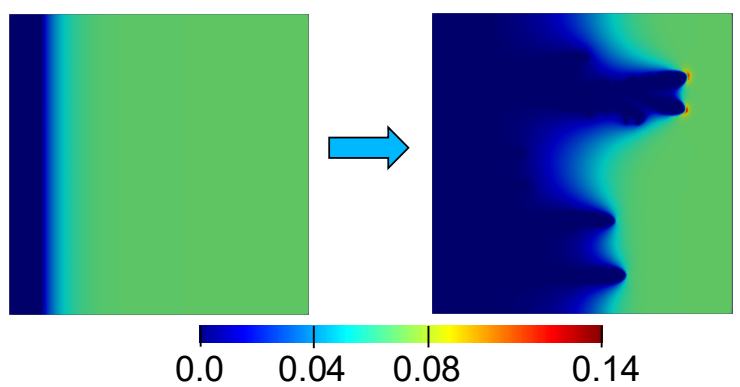

FIG. 4. Correlation between the Li-ion concentration profile and the formation of dendrites. (a) The Li-ion concentration at the interface can be higher (blue, $-0.30 \mathrm{~V}$ applied at $268 \mathrm{~K}$ ) or lower (orange, $-0.44 \mathrm{~V}$ applied at $333 \mathrm{~K}$ ) than that of the electrolyte bulk. (b) dendrite does not form when the interfacial Li-ion concentration is higher than that of the bulk. (c) dendrite forms when the interfacial Li-ion concentration is lower than that of the bulk.

The prediction of the dendrite formation based on the Li-ion concentration profile is shown in Fig. 5(a). The prediction shows a clear pattern where no dendrite is formed when Li electrodeposition takes place at low operating temperature and applied overpotential, while the opposite is true for the high temperature and overpotential. The fact that a high applied overpotential promotes the dendrite formation agrees well with previous simulation ${ }^{17,18,22,28}$ and experimental ${ }^{11,24}$ observations. It is noted that the absence of dendrites at $298 \mathrm{~K}$ under $-0.32 \mathrm{~V}$ overpotential disagrees with the results reported by Hong and Viswanathan ${ }^{17}$, although their simulation conditions were very $\operatorname{similar}(T=300 \mathrm{~K}$ and $\phi=-0.32 \mathrm{~V})$. We verified that the discrepancy originates from the difference in the electrochemical reaction kinetic coefficient values used in the model; our temperature-dependent parameter value differs from the value they used, and we confirmed that modifying the coefficient to match their value led to the same results as reported previously. However, we emphasize that it is not advised to meticulously interpret the threshold temperature/overpotential value for 
dendrite formation since they can shift somewhat based on the parameter values used.

(a)

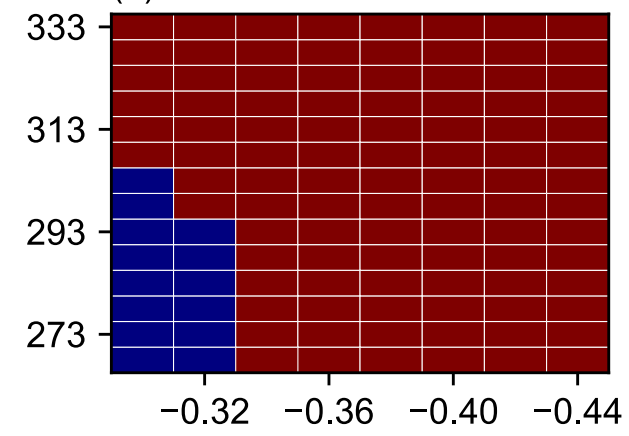

- No dendrite $\square$ Dendrite

(b)

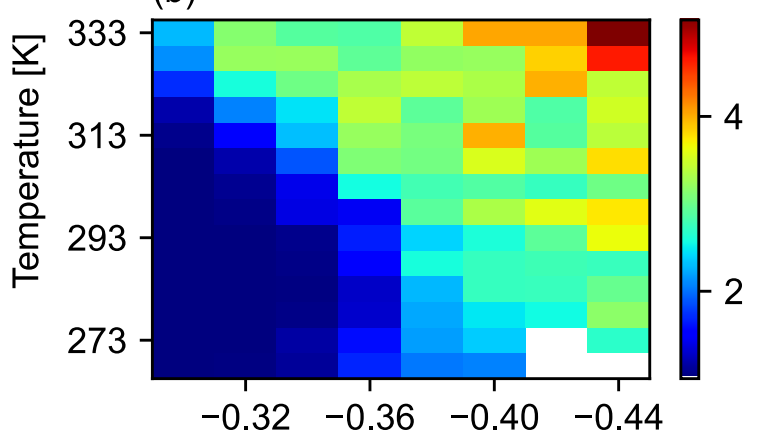

(c)

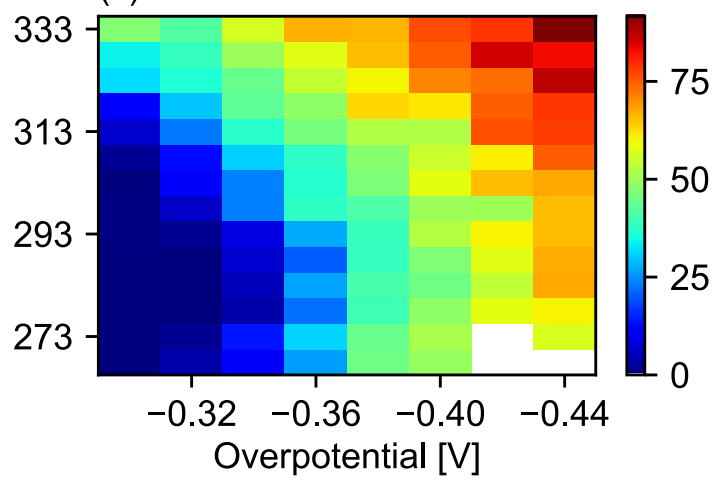

FIG. 5. Surface modulation of the Li metal upon electrodeposition at different overpotentials and temperatures. (a) Prediction of the dendrite formation based on the Li-ion concentration profile early in the electrodeposition process. The tortuosity (b) and dendrite height (c) when the peak Li height reaches $x=150 \mu \mathrm{m}$. White regions in (b) and (c) represent the missing data due to the numerical instability introduced due to the low operating temperature and high magnitude of overpotential.

The tortuosity and dendrite height of the surface are analyzed to verify the validity of the prediction based on the Li-ion concentration profile. It is important to analyze the sur- 
face modulation after a sufficient electrodeposition process to avoid premature assessment. However, it is difficult to also satisfy the condition of having the same amount of Li ions deposited across all simulation conditions; dendrites start to grow almost immediately when both temperature and overpotential are high, leading the dendrite to reach $x=200 \mu \mathrm{m}$ boundary even a relatively small amount of Li ions is deposited. Consequently, the tortuosity and dendrite height are taken when the peak Li height reaches $x=150 \mu \mathrm{m}$, and the results are shown in Fig. 5(b) and (c). The peak height of $150 \mu \mathrm{m}$ is chosen to avoid the tip from getting too close to the cell boundary.

It can be seen that the prediction based on the concentration profile in Fig. 5(a) agrees well with the tortuosity and dendrite height trend shown in Fig. 5(b) and (c). Both tortuosity and dendrite height are low for low temperature and overpotential and high for high temperature and overpotential; a clear pattern can be seen in the color map of Fig. 5(b) and (c), where the color shift from blue to red when traversed from the bottom-left corner to the top-right corner. The gradual change in tortuosity and dendrite height also reveals that there is no sudden "shift" from a dendrite-free to a dendritic regime. Consequently, one can set a heuristic condition for determining the presence of the dendrite on the surface by setting a threshold on tortuosity and dendrite height.

The tortuosity and dendrite height threshold values can be determined if the maximum tortuosity and dendrite height in the dendrite-free regime is lower than those of the dendritic regime. We observed one outlier at $T=268 \mathrm{~K}$ and $\phi=-0.32 \mathrm{~V}$, where the initial Li-ion concentration profile indicated the absence of dendrite upon electrodeposition. Interestingly, the electrodeposition process switched from reaction-limited to transport-limited in this case, resulting in the dendrite formation at a later stage with the final tortuosity and dendrite height of 1.03 and $3.88 \mu \mathrm{m}$, respectively. Except for this outlier, the distribution of the tortuosity and dendrite height in Fig. 5(b) and (c) agreed well with the boundary between the dendrite-free and dendritic regions shown in Fig. 5(a). The maximum tortuosity and dendrite height in the dendrite-free region are 1.012 and $1.93 \mu \mathrm{m}$, respectively. The minimum tortuosity and dendrite height in the dendritic region are 1.015 and $2.07 \mu \mathrm{m}$, respectively, indicating a smooth transition between the dendritic and dendrite-free regions. Therefore, we set a threshold where the surfaces with the tortuosity larger than 1.014 and the dendrite height higher than $2.05 \mu \mathrm{m}$ are considered to have dendrites. These thresholds are used to determine the onset condition where the dendrites start to form on the surface. 


\section{B. Influence of temperature and overpotential on electrodeposition rate and}

surface modulation

The influence of overpotential and temperature on the electrodeposition rate, tortuosity and dendrite height are compared next. The analysis is carried out using the snapshot taken when the average height of the Li electrode is $55 \mu \mathrm{m}$, ensuring that a systematic comparison is made when the same amount of $\mathrm{Li}$ is deposited. A conservative height of $55 \mathrm{\mu m}$ is chosen to ensure that dendrites do not reach the $x=200 \mu \mathrm{m}$ boundary even for the high temperature and overpotential cases where dendrites form almost immediately. The time it takes to reach the average height of $55 \mu \mathrm{m}$, tortuosity and dendrite height as a function of overpotential and temperature are shown in Fig. 6.
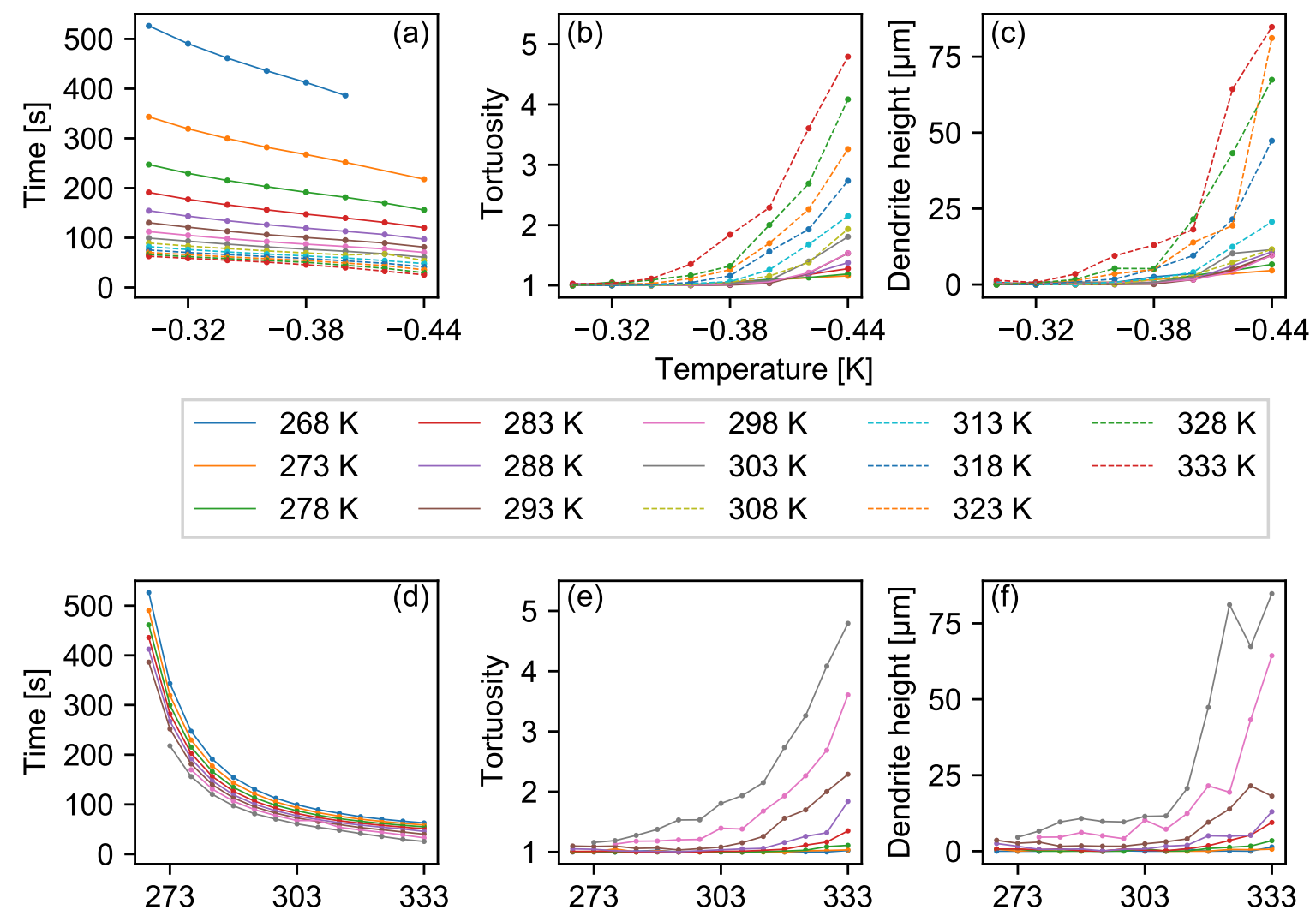

$313 \mathrm{~K}$
$318 \mathrm{~K}$
$323 \mathrm{~K}$
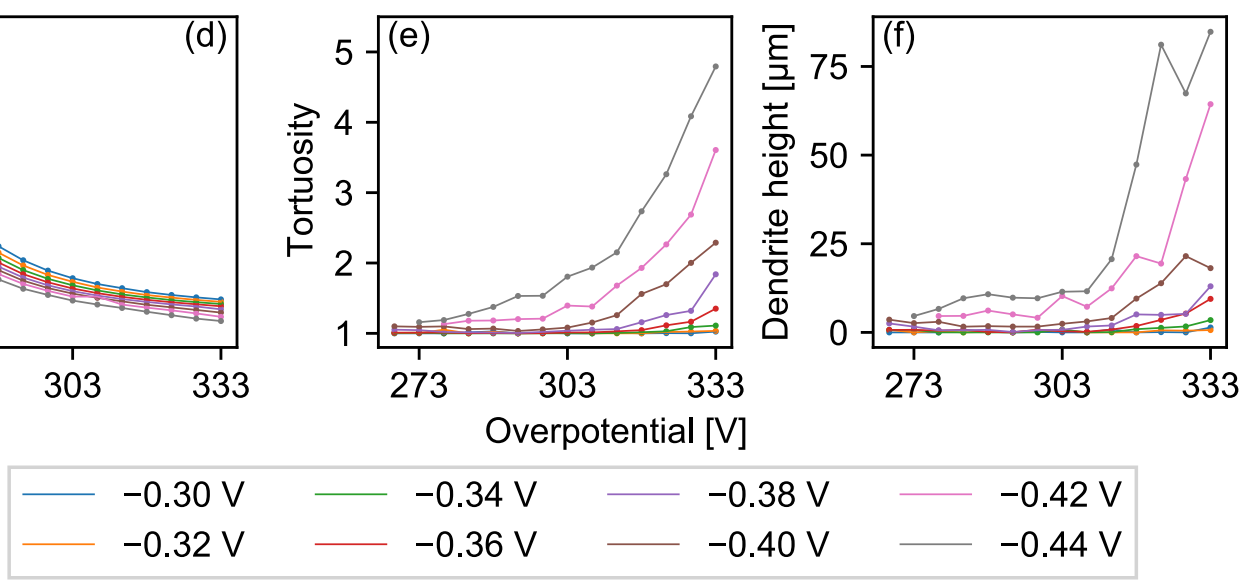

FIG. 6. Influence of overpotential and temperature on $(\mathrm{a}, \mathrm{d})$ the time it takes to reach the average Li height of $55 \mu \mathrm{m}$, (b, e) tortuosity of the surface and $(\mathrm{c}, \mathrm{f})$ dendrite height when the average Li height is $55 \mu \mathrm{m}$. 
Figure 6(a) and (d) show a clear correlation between the time it takes to reach the average height of $55 \mu \mathrm{m}$ and both overpotential and temperature. The elapsed time decreases linearly as the magnitude of the applied overpotential is increased, indicating that the electrodeposition rate (or electric current), a reciprocal of the elapsed time, is proportional to the applied overpotential. Therefore, a direct comparison can be made between galvanostatic and potentiostatic measurement patterns for varying overpotential/current at fixed temperature since they are linearly proportional. On the other hand, the elapsed time is inversely proportional to temperature and displays a sharp decay as the temperature is increased (Fig. 6(d)), which highlights an implication in making a direct comparison between the temperature dependence of galvanostatic and potentiostatic electrodeposition. The induced electric current is proportional to temperature when the overpotential is fixed. In other words, increasing the temperature under potentiostatic conditions will increase the induced current while it decreases the induced overpotential under galvanostatic conditions. The interpretation drawn from our simulations agrees well with the experimental observations by Yan et al. ${ }^{20}$ where the induced overpotential in the galvanostatic setting is decreased when the temperature is increased. Therefore, it is important to consider both galvanostatic and potentiostatic processes to gain a deeper understanding of the Li nucleation mechanism in future studies. ${ }^{24}$

Both tortuosity and dendrite height show a nonlinear growth as a function of applied overpotential (Fig. 6(b) and (c)); both tortuosity and height increase rapidly after the dendrite starts to form, and the magnitude of the onset potential becomes higher as the temperature is increased. A similar pattern is observed for the temperature dependence (Fig. 6(e) and (f)), where both tortuosity and dendrite height grow rapidly after the onset temperature, and the onset point becomes lower as the magnitude of the applied overpotential is increased. It is also found that tortuosity and dendrite height show a similar temperatureand overpotential dependency, which allows one to estimate the evolution of tortuosity or dendrite height based on the evolution pattern of another.

The discussion in this section was limited to the case where the average Li height is $55 \mu \mathrm{m}$ for a systematic assessment of the surface modulation. While the comparison is made on the same average Li height as the same height corresponds to the same amount of Li deposited on the electrode, the scope was limited to a single Li height. The evolution of tortuosity and dendrite height as a function of average Li height is shown in Fig. 7, where the curves are 
terminated when the $\mathrm{Li}$ peak reaches the height of $150 \mu \mathrm{m}$. It can be seen that the average Li height at the onset of the dendrite formation (in terms of both tortuosity and dendrite height) becomes lower as the magnitude of the overpotential and temperature are increased, agreeing with the patterns observed so far. Additionally, both tortuosity and dendrite height increase almost linearly with respect to the average Li height beyond the onset point, and their slopes are quite similar across the entire range of overpotential and temperature. The linear increase of tortuosity and dendrite height indicates that it is critical to compare the onset point of the dendrite formation since they continue to grow at a rather predictable rate afterward.

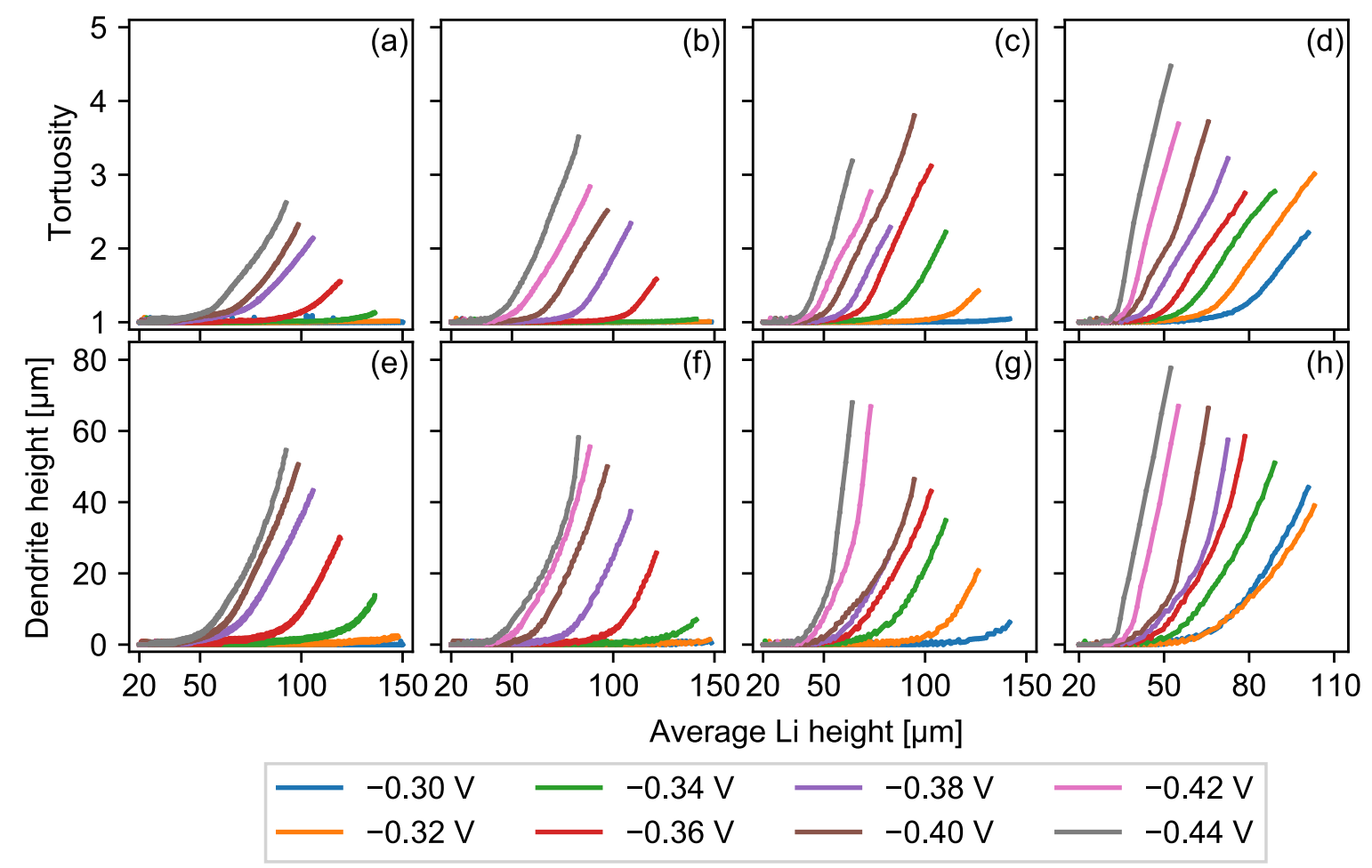

FIG. 7. Evolution of $(\mathrm{a}-\mathrm{d})$ tortuosity and $(\mathrm{e}-\mathrm{f})$ dendrite height as a function of average Li height at (a, e) $273 \mathrm{~K},(\mathrm{~b}, \mathrm{f}) 293 \mathrm{~K},(\mathrm{c}, \mathrm{g}) 313 \mathrm{~K}$ and (d, h) $333 \mathrm{~K}$. Tortuosity and dendrite height are shown until the peak Li height reaches $150 \mu \mathrm{m}$. 


\section{Influence of temperature and overpotential on the onset point of dendrite formation}

We have established threshold criteria on tortuosity and dendrite height to determine the onset point at which dendrites start to form and demonstrated that it is critical to determine the onset point. It is shown in Fig. 8 that the onset of the dendrite formation decreases almost monotonically as the magnitude of applied overpotential is increased for all temperatures. The pattern makes intuitive sense as applying a higher overpotential to drive the electrodeposition is more likely to introduce the inhomogeneity on the surface while allowing less time for the surface ions to diffuse along the surface. Therefore, it is desirable to reduce the magnitude of overpotential to suppress dendrite formation.

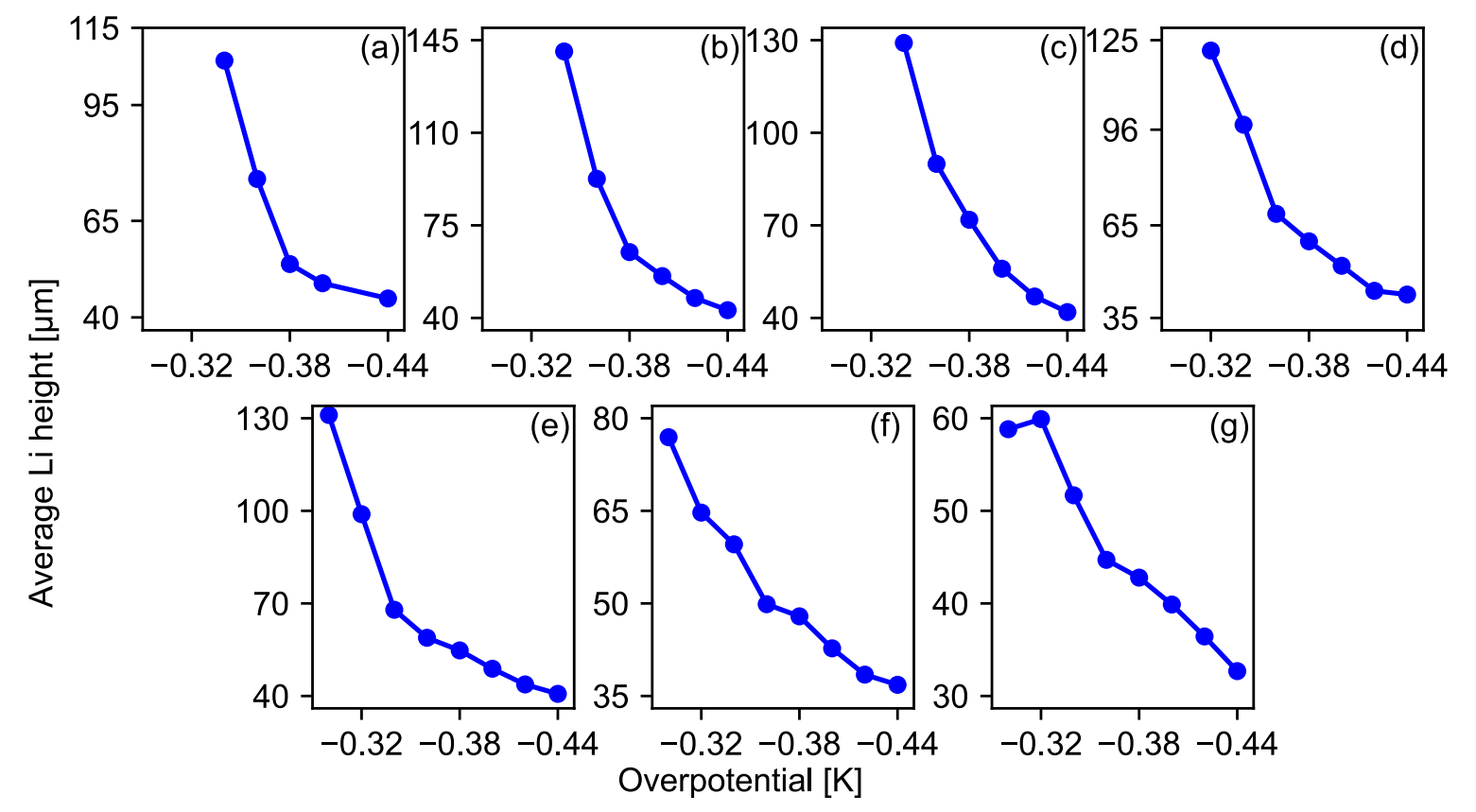

FIG. 8. Average height of Li electrode at the onset of dendrite formation when temperature is (a) $273 \mathrm{~K}$, (b) $283 \mathrm{~K}$, (c) $293 \mathrm{~K}$, (d) $303 \mathrm{~K}$, (e) $313 \mathrm{~K}$, (f) $323 \mathrm{~K}$ and (g) $333 \mathrm{~K}$.

The temperature dependence of the onset point, on the other hand, is not as straightforward as the overpotential. As shown in Fig. 9, there is no monotonic increase or decrease in onset point as a function of temperature. Note that the temperature dependency of the onset point is not reported for $\phi=-0.30 \mathrm{~V}$ and $-0.32 \mathrm{~V}$ as dendrites do not form at low temperatures. Despite some fluctuations in the pattern, it can be seen in Fig. 9 that the onset point increases as the temperature increases in the low-temperature region and decreases 
in the high-temperature region. The temperature at which the onset point reaches its peak height is the optimal operating temperature that inhibits the formation of dendrites. The optimal temperature gradually shifts from $283 \mathrm{~K}$ to $298 \mathrm{~K}$ as the applied overpotential is increased from $-0.34 \mathrm{~V}$ to $-0.42 \mathrm{~V}$. A slight anomaly of a sudden decrease in the optimal temperature to $273 \mathrm{~K}$ is observed when the overpotential is $-0.44 \mathrm{~V}$ (Fig. 9(f)). However, the difference between the highest average height at $273 \mathrm{~K}$ and the second-highest average height at $293 \mathrm{~K}$ is sufficiently low to consider them to be within the error margin.

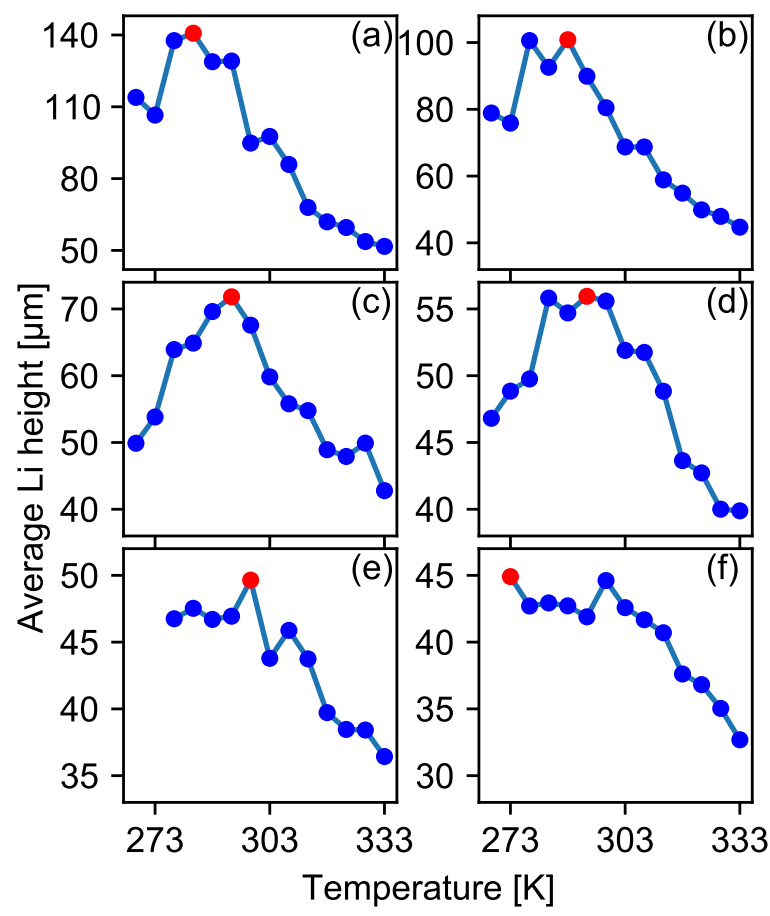

FIG. 9. Average height of Li electrode at the onset of dendrite formation when the applied overpotential is (a) $-0.34 \mathrm{~V}$, (b) $-0.36 \mathrm{~V},(\mathrm{c})-0.38 \mathrm{~V},(\mathrm{~d})-0.40 \mathrm{~V},(\mathrm{e})-0.42 \mathrm{~V}$ and (f) $-0.44 \mathrm{~V}$. Red points indicate the temperature at which the average Li height reaches its maximum value.

The optimal operating conditions do not exceed room temperature regardless of the applied overpotential value. Further increase in temperature lowers the onset point of the dendrite formation, which implies that increasing the temperature beyond room temperature promotes the dendrite formation. Such a pattern contrasts the previous reports where elevating the temperature is found to be beneficial for dendrite suppression. ${ }^{20,25}$ As pointed out previously, the difference stems from the fact that these studies are carried out under galvanostatic conditions where the increase in temperature lowers the overpotential across the interface. In contrast, the potentiostatic simulations are carried out in this work. The 
elevated temperature causes the induced current to increase, making it difficult to compare the dendrite suppression observed in galvanostatic measurements directly. Such discrepancy highlights that care must be given when comparing the thermal effects observed in galvanostatic and potentiostatic conditions.

\section{CONCLUSIONS}

A nonlinear phase-field model that integrates thermal effects is presented. The thermal

effect was reflected via the use of temperature-dependent values of electrode and electrolyte conductivity, surface tension, exchange current density and Li-ion diffusivity in the electrolyte. The correlation between the Li-ion concentration profile at the interface and the dendrite formation is verified, and the correlation is used to set thresholds on surface tortuosity and dendrite height that defines the onset condition for dendrite formation. It was found that elevating the temperature increases the induced current and accelerates the electrodeposition process. The increase in temperature and overpotential promotes dendrite formation. The onset point for the dendrite formation is a crucial factor to consider since the rate of dendrite growth with respect to the amount of deposited Li is similar across all temperatures and overpotential. It was determined that increasing the overpotential lowers the onset point. The temperature dependence of the onset point was not linear, and the optimal operating temperature is observed to increase as the magnitude of overpotential is increased. A deeper insight into the underlying mechanism of dendrite formation and growth can be gained via combined potentiostatic and galvanostatic investigation using a phase-field model that incorporates the internal heat generation, convection and radiation in the future.

\section{ACKNOWLEDGMENTS}

This research was supported by the MOTIE (Ministry of Trade, Industry, and Energy) in Korea, under the Fostering Global Talents for Innovative Growth Program related to Robotics (P0008749) supervised by the Korea Institute for Advancement of Technology (KIAT) and by Korea Institute of Energy Technology Evaluation and Planning (KETEP) grant funded by the Korea government (MOTIE) (20202020800030, Development of Smart 
Hybrid Envelope Systems for Zero Energy Buildings through Holistic Performance Test and Evaluation Methods and Fields Verifications). The authors acknowledge support from the Stakeholder Initiative project CANADA under the European Union's Horizon 2020 research and innovation programme under Grant Agreement No. 957189 (BIG-MAP).

\section{REFERENCES}

${ }^{1}$ L. He, Q. Sun, L. Lu, and S. Adams, "Understanding and Preventing Dendrite Growth in Lithium Metal Batteries," ACS Applied Materials and Interfaces 13, 34320-34331 (2021). ${ }^{2}$ Q. Wu, Y. Yang, Z. Chen, Q. Su, S. Huang, D. Song, C. Zhu, R. Ma, and C. Li, "DendriteFree Solid-State Li Metal Batteries Enabled by Bifunctional Polymer Gel Electrolytes," ACS Applied Energy Materials (2021), 10.1021/acsaem.1c01634.

${ }^{3}$ P. Hundekar, R. Jain, A. S. Lakhnot, and N. Koratkar, "Recent advances in the mitigation of dendrites in lithium-metal batteries," Journal of Applied Physics 128 (2020), 10.1063/5.0015099.

${ }^{4}$ X. Xu, S. Wang, H. Wang, B. Xu, C. Hu, Y. Jin, J. Liu, and H. Yan, "The suppression of lithium dendrite growth in lithium sulfur batteries: A review," Journal of Energy Storage 13, 387-400 (2017).

${ }^{5}$ J. Wang, S. Yi, J. Liu, S. Sun, Y. Liu, D. Yang, K. Xi, G. Gao, A. Abdelkader, W. Yan, S. Ding, and R. V. Kumar, "Suppressing the Shuttle Effect and Dendrite Growth in Lithium-Sulfur Batteries," ACS Nano 14, 9819-9831 (2020).

${ }^{6}$ R. Demir-Cakan, "Introduction to Rechargeable Lithium-Sulfur Batteries," in Li-S Batteries (WORLD SCIENTIFIC (EUROPE), 2017) pp. 1-30.

${ }^{7}$ G. Girishkumar, B. McCloskey, A. C. Luntz, S. Swanson, and W. Wilcke, "Lithium-Air Battery: Promise and Challenges," The Journal of Physical Chemistry Letters 1, 2193$2203(2010)$.

${ }^{8}$ X. Zou, Q. Lu, Y. Zhong, K. Liao, W. Zhou, and Z. Shao, "Flexible, Flame-Resistant, and Dendrite-Impermeable Gel-Polymer Electrolyte for Li-O2/Air Batteries Workable Under Hurdle Conditions," Small 14, 1-10 (2018).

${ }^{9} \mathrm{~N}$. Imanishi and O. Yamamoto, "Perspectives and challenges of rechargeable lithium-air batteries," Materials Today Advances 4, 100031 (2019). 
${ }^{10}$ H. Yu, D. Liu, X. Feng, and Y. Zhang, "Mini review: Recent advances on flexible rechargeable Li-air batteries," Energy and Fuels 35, 4751-4761 (2021).

${ }^{11}$ Y. Liu, X. Xu, M. Sadd, O. O. Kapitanova, V. A. Krivchenko, J. Ban, J. Wang, X. Jiao, Z. Song, J. Song, S. Xiong, and A. Matic, "Insight into the Critical Role of Exchange Current Density on Electrodeposition Behavior of Lithium Metal," Advanced Science 8, 2003301 (2021).

${ }^{12}$ W. Mu, X. Liu, Z. Wen, and L. Liu, "Numerical simulation of the factors affecting the growth of lithium dendrites," Journal of Energy Storage 26, 100921 (2019).

${ }^{13}$ S. Rajendran, Z. Tang, A. George, A. Cannon, C. Neumann, A. Sawas, E. Ryan, A. Turchanin, and L. M. R. Arava, "Inhibition of Lithium Dendrite Formation in Lithium Metal Batteries via Regulated Cation Transport through Ultrathin Sub-Nanometer Porous Carbon Nanomembranes," Advanced Energy Materials 11, 2100666 (2021).

${ }^{14}$ W. Xu, J. Wang, F. Ding, X. Chen, E. Nasybulin, Y. Zhang, and J. G. Zhang, "Lithium metal anodes for rechargeable batteries," Energy and Environmental Science 7, 513-537 (2014).

${ }^{15}$ H. H. Yan, Y. H. Bie, X. Y. Cui, G. P. Xiong, and L. Chen, "A computational investigation of thermal effect on lithium dendrite growth," Energy Conversion and Management 161, 193-204 (2018).

${ }^{16}$ Z. Hong and V. Viswanathan, "Prospect of Thermal Shock Induced Healing of Lithium Dendrite," ACS Energy Letters 4, 1012-1019 (2019).

${ }^{17}$ Z. Hong and V. Viswanathan, "Phase-Field Simulations of Lithium Dendrite Growth with Open-Source Software," ACS Energy Letters 3, 1737-1743 (2018).

${ }^{18}$ L. Chen, H. W. Zhang, L. Y. Liang, Z. Liu, Y. Qi, P. Lu, J. Chen, and L.-Q. Chen, "Modulation of dendritic patterns during electrodeposition: A nonlinear phase-field model," Journal of Power Sources 300, 376-385 (2015).

${ }^{19}$ D. A. Cogswell, "Quantitative phase-field modeling of dendritic electrodeposition," Physical Review E 92, 011301 (2015).

${ }^{20}$ K. Yan, J. Wang, S. Zhao, D. Zhou, B. Sun, Y. Cui, and G. Wang, "TemperatureDependent Nucleation and Growth of Dendrite-Free Lithium Metal Anodes," Angewandte Chemie International Edition 58, 11364-11368 (2019).

${ }^{21}$ S. Wang, K. Rafiz, J. Liu, Y. Jin, and J. Y. S. Lin, "Effects of lithium dendrites on thermal runaway and gassing of $\mathrm{LiFePO}_{4}$ batteries," Sustainable Energy \& Fuels 4, $2342-$ 
$2351(2020)$.

${ }^{22}$ X. Gao, Y.-N. Zhou, D. Han, J. Zhou, D. Zhou, W. Tang, and J. B. Goodenough, "Thermodynamic Understanding of Li-Dendrite Formation," Joule 4, 1864-1879 (2020).

${ }^{23}$ C. T. Love, O. A. Baturina, and K. E. Swider-Lyons, "Observation of Lithium Dendrites at Ambient Temperature and Below," ECS Electrochemistry Letters 4, A24-A27 (2014).

${ }^{24}$ A. Pei, G. Zheng, F. Shi, Y. Li, and Y. Cui, "Nanoscale Nucleation and Growth of Electrodeposited Lithium Metal," Nano Letters 17, 1132-1139 (2017).

${ }^{25}$ L. Li, S. Basu, Y. Wang, Z. Chen, P. Hundekar, B. Wang, J. Shi, Y. Shi, S. Narayanan, and N. Koratkar, "Self-heating-induced healing of lithium dendrites," Science 359, 1513-1516 (2018).

${ }^{26}$ B. Stinner, Derivation and Analysis of a Phase Field Model for Alloy Solidification, Ph.D. thesis, Universität Regensburg (2005).

${ }^{27}$ S. L. Wang, R. F. Sekerka, A. A. Wheeler, B. T. Murray, S. R. Coriell, R. J. Braun, and G. B. McFadden, "Thermodynamically-consistent phase-field models for solidification," Physica D: Nonlinear Phenomena 69, 189-200 (1993).

${ }^{28}$ L. Liang, Y. Qi, F. Xue, S. Bhattacharya, S. J. Harris, and L. Q. Chen, "Nonlinear phasefield model for electrode-electrolyte interface evolution," Physical Review E - Statistical, Nonlinear, and Soft Matter Physics 86, 1-5 (2012).

${ }^{29}$ C.-H. Chen and C.-W. Pao, "Phase-field study of dendritic morphology in lithium metal batteries," Journal of Power Sources 484, 229203 (2021).

${ }^{30}$ K. Wang, Y. Xiao, P. Pei, X. Liu, and Y. Wang, "A Phase-Field Model of Dendrite Growth of Electrodeposited Zinc," Journal of The Electrochemical Society 166, D389-D394 (2019).

${ }^{31}$ L. Gao and Z. Guo, "Phase-field simulation of Li dendrites with multiple parameters influence," Computational Materials Science 183, 109919 (2020).

${ }^{32}$ https : //www . phasetree.ai.

${ }^{33}$ A. Jana, S. I. Woo, K. S. N. Vikrant, and R. E. García, "Electrochemomechanics of lithium dendrite growth," Energy \& Environmental Science 12, 3595-3607 (2019).

${ }^{34}$ M. Hess, "Temperature-Dependence of the Solid-Electrolyte Interphase Overpotential: Part I. Two Parallel Mechanisms, One Phase Transition," Journal of The Electrochemical Society 165, A323-A332 (2018).

${ }^{35}$ L. O. Valøen and J. N. Reimers, "Transport Properties of LiPF ${ }_{6}$-Based Li-Ion Battery Electrolytes," Journal of The Electrochemical Society 152, A882 (2005). 
${ }^{36}$ T. C. Chi, "Electrical resistivity of alkali elements," Journal of Physical and Chemical Reference Data 8, 339-438 (1979).

${ }^{37} \mathrm{~W}$. Tyson and W. Miller, "Surface free energies of solid metals: Estimation from liquid surface tension measurements," Surface Science 62, 267-276 (1977).

${ }^{38}$ M. W. Verbrugge and B. J. Koch, "Microelectrode Study of the Lithium/Propylene Carbonate Interface: Temperature and Concentration Dependence of Physicochemical Parameters," Journal of The Electrochemical Society 141, 3053-3059 (1994).

${ }^{39}$ C. Monroe and J. Newman, "Dendrite Growth in Lithium/Polymer Systems," Journal of The Electrochemical Society 150, A1377 (2003).

${ }^{40}$ H. Sano, H. Sakaebe, H. Senoh, and H. Matsumoto, "Effect of Current Density on Morphology of Lithium Electrodeposited in Ionic Liquid-Based Electrolytes," Journal of The Electrochemical Society 161, A1236-A1240 (2014).

${ }^{41}$ H.-W. Zhang, Z. Liu, L. Liang, L. Chen, Y. Qi, S. J. Harris, P. Lu, and L.-Q. Chen, "Understanding and Predicting the Lithium Dendrite Formation in Li-Ion Batteries: Phase Field Model," ECS Transactions 61, 1-9 (2014).

${ }^{42}$ L. Frenck, G. K. Sethi, J. A. Maslyn, and N. P. Balsara, "Factors That Control the Formation of Dendrites and Other Morphologies on Lithium Metal Anodes," Frontiers in Energy Research 7 (2019), 10.3389/fenrg.2019.00115.

${ }^{43}$ M. Jäckle, K. Helmbrecht, M. Smits, D. Stottmeister, and A. Groß, "Self-diffusion barriers: Possible descriptors for dendrite growth in batteries?" Energy and Environmental Science 11, 3400-3407 (2018). 